\title{
COMPARISON OF TURBINOPLASTY SURGERY EFFICACY IN PATIENTS WITH AND WITHOUT ALLERGIC RHINITIS AT MGMMC, JAMSHEDPUR
}

\section{ENT}

\section{Dr. Devendra Jee}

M.S. (ENT), Associate Professor and Head of Department, Department of ENT, MGM Medical College \& Hospital, Jamshedpur, Jharkhand.

\begin{tabular}{ll}
\hline $\begin{array}{l}\text { Dr. Rohit Kumar } \\
\text { Jha }\end{array}$ & $\begin{array}{l}\text { M.S. (ENT), Assistant Professor, Department of ENT, MGM Medical College \& Hospital, } \\
\text { Jamshedpur, Jharkhand. }\end{array}$ \\
\hline $\begin{array}{l}\text { Dr. Bhimsen } \\
\text { Hansda* }\end{array}$ & M.S. (ENT), Assistant Professor, Department of ENT, MGM Medical College \& Hospital, \\
\hline
\end{tabular}

Dr. Debarshi Jana

Young Scientist (DST),Institute of Post-Graduate Medical Education and Research, A.J.C. Bose Road, Kolkata-700020, West Bengal, India.

\section{ABSTRACT}

INTRODUCTION: Turbinoplasty is a procedure that aims to reduce the size of the inferior turbinate through exuberant bone removal with high mucosal preservation. The procedure is recommended for patients with or without allergic rhinitis and those showing irreversible hypertrophy of inferior turbinates.

OBJECTIVE: To evaluate the efficacy of inferior turbinoplasty for obstructive and non-obstructive symptoms in patients with or without allergic rhinitis.

METHODS: Prospective study with 57 patients who underwent inferior turbinoplasty. They were evaluated for nasal obstruction, snoring, facial pressure, smell alterations, sneezing, nasal itching and runny nose symptoms, surgery time, and intraoperative bleeding. The last evaluation took place three months after surgery.

RESULTS:Thirty-nine patients with allergic rhinitis and 18 without were assessed. Ninety days after surgery, $94.7 \%$ of patients showed degrees IV and V of breathing improvement; $89.5 \%$ showed moderate or complete improvement in snoring; all patients showed smell improvement (only one showed moderate improvement; all the others had full improvement); $95.5 \%$ experienced complete facial pressure improvement; and $89.7 \%$ showed moderate to complete improvement in nasal itching and runny nose symptoms, as well as in sneezing.

CONCLUSION: The efficacy of inferior turbinoplasty was confirmed not only for obstructive symptoms, but also for non-obstructive symptoms in patients with and without allergic rhinitis.

\section{KEYWORDS}

Turbinates; Rhinitis; Olfaction disorders; Smell; Snoring; Nasal obstruction

\section{INTRODUCTION}

Nasal obstruction affects approximately $25 \%$ of the population. It is a symptom that can affect people of all ages and ethnicities. It has some degree of morbidity, which varies with the severity and cause of nasal obstruction. The main causes are: septal deviation, inferior and medial turbinate hypertrophy, nasal polyps, and pharyngeal tonsil hypertrophy. Of all of these alterations, inferior turbinate hypertrophy is the most common cause of nasal obstruction. ${ }^{2}$ The leading causes of inferior turbinate hypertrophy are allergic rhinitis, vasomotor rhinitis, and septal deviation (compensatory hypertrophy). Bilateral nasal obstruction usually occurs with mucosal disease. When associated with watery rhinorrhea, sneezing, and nasal itching, it is characteristic of nasal mucosa inflammatory edema, especially of allergic nature.

Rhinitis is the inflammation of the nasal mucosal lining, characterized by the presence of one or more symptoms: nasal congestion, rhinorrhea, sneezing, itching, and hyposmia. Nasal obstruction is one of the most inconvenient symptoms for the patient. The diagnosis of allergic rhinitis includes personal and family history of atopy, physical examination, and complementary exams. The diagnosis is essentially clinical, taking into account the association of the several symptoms. The most important complementary exams in the diagnosis of allergic rhinitis, for both specificity and sensitivity, are immediate hypersensitivity skin prick test (SPT) using the puncture technique and evaluation of serum levels of allergen-specific IgE

The determination of specific IgE in vitro may be accomplished by several enzyme immunoassay methods, and more recently, by immunofluorescence. Specific IgE assay in vitro for individual allergens, when performed with standardized antigens and adequate technique, has operational characteristics (sensitivity and specificity) that are similar to those of the skin prick test: sensitivity of $89 \%$ and specificity of $91 \%$. Treatment includes both non-pharmacological environmental control - and pharmacological measures. The latter are based on antihistamines, decongestants, topical and systemic corticoids, and other medications such as ipratropium bromide, chromoglycate disodium, and anti-leukotrienes.
Immunotherapy and the use of saline solution for nasal irrigation are other choices. Modern pharmacology offers many options for clinical treatment of inferior turbinate hypertrophy, whatever the source is. However, although still a controversial issue, most authors agree that when clinical treatment is not sufficient to provide adequate nasal airways, surgical treatment should be indicated.

Surgical treatment of allergic rhinitis refractory to clinical treatment is directed to the inferior turbinates and aims to increase the nasal cavity without altering nasal physiology. The search for effective nasal turbinate treatment has stimulated the surgical skill of rhinologists for over 100 years

Surgical procedures that aim to reduce mucosal or boney hypertrophy of the inferior nasal turbinate, or both, include: corticosteroid infiltration, lateral dislocation of the nasal turbinate, partial turbinectomy, lower turbinoplasty, cryosurgery, laser vaporization, and radiofrequency. Turbinoplasty is a procedure aimed at reducing the size of the inferior turbinate through exuberant bone removal and meatal surface removal with greater mucosal preservation. It allows reduction of the turbinate volume, while maintaining the physiological functions of the mucosa. It is performed through an incision along the border of the turbinates, detachment and exposure of the bone surface, followed by bone and redundant mucosa removal, covering the remaining bone with the excess mucosa. Nasal turbinate surgery is one of the most frequently performed procedures in otorhino laryngologists' daily practice, recognized as an effective treatment for nasal obstruction secondary to hypertrophic rhinitis

\section{AIM OF THE STUDY}

The aim of this study is to assess the efficacy of inferior turbinoplasty surgery on obstructive and non-obstructive symptoms in patients with and without allergic rhinitis.

\section{MATERIALAND METHODS}

A prospective study is done over a period of 1 year at department of ENT, MGM Medical College and Hospital, Jamshedpur, Jharkhand. 
This study involved 57 patients treated on an outpatient basis, prospectively evaluated and submitted to inferior turbinoplasty surgery. All patients had inferior turbinate hypertrophy and nasal obstruction refractory to medical treatment with at least two months of topical nasal corticosteroid and systemic antihistamine use, and no other nasal conditions, such as septal deviation, middle concha bullosa, or hypertrophic middle turbinate.

Patients were divided into two groups: group I - patients with nasal obstruction and symptoms of rhinorrhea, sneezing, and itchy nose were considered as having allergic rhinitis; and group II - patients with nasal obstruction without the other described symptoms - were considered without allergic rhinitis.

Specific IgE assays were performed on the blood of all patients to aeroallergens, carried out preoperatively in vitro for detection of allergic rhinitis; the tested allergens, which are common in the local environment, are grasses, dust mites, pollen, fungi, and dog and cat epithelium

All patients were operated on by the same surgeon, under local anesthesia and sedation.

The following inclusion criteria were used: patients with chronic nasal obstruction, without improvement after standard medications (systemic and topical corticosteroids and systemic antihistamines) for at least two months; aged between 14 and 70 years; willing to participate in the study and to answer the protocol questions; available to return for reassessment seven, 30 , and 90 days after surgery; patients with irreversible hypertrophy of the inferior turbinate; patients divided into groups with and without allergic rhinitis, based on the symptoms of itching, sneezing and rhinorrhea, in addition to nasal obstruction.

Patients with any significant anatomical alterations (apart from inferior turbinate hypertrophy) that generated nasal obstruction (septal deviation, middle concha bullosa, nasal valve alterations, nasal tumors of any nasal-sinus origin, retro-nasal or paranasal masses, choanal imperforation, septal perforation, unciform process abnormalities, nasal polyps, adenoid hypertrophy); those who showed improvement after clinical treatment; pregnant women; those who could not undergo the turbinoplasty due to clinical status; and those not willing to participate and answer the protocol questions were excluded.

Patients were evaluated preoperatively regarding gender; age; intensity of nasal obstruction (mild, moderate, severe); presence or absence of snoring; presence or absence of facial pressure; presence or absence of smell alterations; presence or absence of sneezing, itching, and rhinorrhea. Time of operation was assessed transoperatively after turbinate infiltration and placing of cottonoid patties with vasoconstrictor solution until the end of the procedure (0-5 $\mathrm{min}, 5-10$ min, 10-15 min, 15-20 min, over $20 \mathrm{~min}$ ) and intraoperative bleeding (+/IV;++/IV;+++/IV;++++/IV).

At seven days postoperatively, breathing improvement grade (grades 1-5) was assessed. Thirty days after surgery, breathing improvement grade was assessed again. Finally, 90 days after surgery, breathing improvement grade was assessed, as well as olfaction improvement grade (no improvement, moderate improvement, total improvement); facial pressure improvement grade (no improvement, moderate improvement, total improvement); snoring improvement grade (no improvement, moderate improvement, total improvement) and sneezing, itching, and rhinorrhea improvement grade (no improvement, moderate improvement, and total improvement).

The turbinoplasty surgery technique consisted of the following: incision in the center of the inferior turbinate in its horizontal extension (Fig. 1); detachment of the entire mucosa above the incision, creating a mucosal flap; next, the turbinate was incised in the anterior-posterior direction using turbinectomy scissors and then one of the scissors blades was directed to the mucosa detached from the bone in the upper part of the turbinate, while the other was directed to the inferior meatus (Fig. 2), thereby removing most of the bone and all its lateral mucosa; the medial mucosa was removed only below the incision, as the mucosa above was used to cover the bony remnant, thereby removing $50 \%$ of the medial mucosa (septal surface), $100 \%$ of the lateral mucosa (meatal surface), and $70 \%$ of the bony turbinate (Fig. 3). Subsequently, the bone spicules were removed with a chisel to reduce the bulging of the remaining turbinate (Fig. 4), and then electrocautery was used to cauterize occasional bleeding spots, particularly in the turbinate tail.

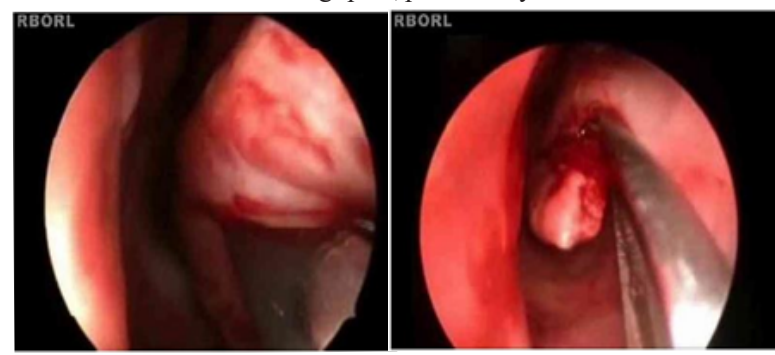

Figure 1 Incision line on the inferior turbinate (endoscopic view). Figure 2 Direction of the turbinectomy scissors blades.

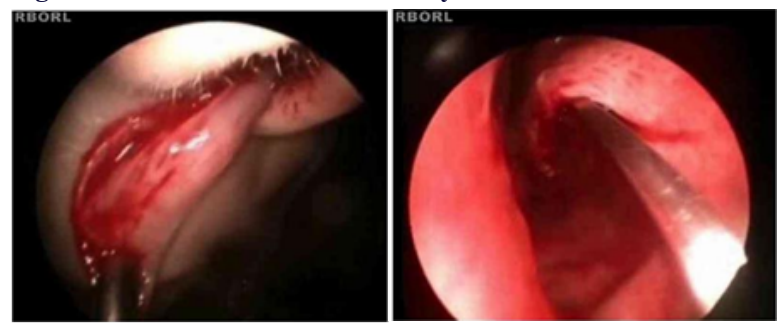

Figure 3 Removal of resected turbinate.

Figure 4 Removal of bony spicule using a chisel.

After that, the patients were transferred to the recovery room and discharged in approximately $4 \mathrm{~h}$ after the surgery, without nasal packing. They were instructed to avoid efforts or blowing the nose on the first postoperative days and were warned that a small amount of bleeding is common. On the second day after surgery, they started to perform nasal irrigation with $0.9 \%$ saline solution to remove the crusts. Saline solution nasal spray could also be used for this purpose. Nasal washing is an important operation step for rapid improvement of nasal obstruction and cleaning

Statistical analysis of pre-, trans- and postoperative parameters was carried out, comparing the differences between groups I and II. The significance level was set at $p<0.05$, using the chi-squared and Fisher's exact tests.

\section{RESULTS}

A total of 57 patients were included in this study, of whom 39 were diagnosed with allergic rhinitis (group I) and 18 without (group II). Preoperative data are shown in Table 1. The sample included 30 male individuals, 20 in group I and ten in group II; and 27 females, with 19 in group I and eight in group II.

Table 1 Preoperative parameters.

\begin{tabular}{|c|c|c|c|c|c|c|c|c|c|c|c|c|}
\hline & \multicolumn{2}{|c|}{ Gender } & \multicolumn{2}{|c|}{ Nasal obstruction } & \multicolumn{2}{|c|}{ Snoring } & \multicolumn{2}{|c|}{ Facial pressure } & \multicolumn{2}{|c|}{ Olfaction alterations } & \multicolumn{2}{|c|}{ IgE } \\
\hline & Male & Female & Moderate & Severe & Present & Absent & Present & Absent & Present & Absent & Positive & Negative \\
\hline \multicolumn{13}{|c|}{ With allergic rhinitis } \\
\hline$n$ & 20 & 19 & 24 & 15 & 29 & 10 & 16 & 23 & 13 & 26 & 29 & 10 \\
\hline$\%$ & 51.3 & 48.7 & 61.5 & 38.5 & 74.4 & 25.6 & 41.0 & 59.0 & 33.3 & 66.7 & 74.4 & 25.6 \\
\hline \multicolumn{13}{|c|}{ Without allergic rhinitis } \\
\hline$n$ & 10 & 8 & 14 & 4 & 9 & 9 & 6 & 12 & 2 & 16 & 2 & 16 \\
\hline$\%$ & 55.6 & 44.4 & 77.8 & 22.2 & $50 \%$ & $50 \%$ & 33.3 & 66.7 & 11.1 & 88.9 & 11.1 & 88.9 \\
\hline Total & 30 & 27 & 38 & 19 & 38 & 19 & 22 & 35 & 15 & 42 & 31 & 26 \\
\hline$p$-Value & \multicolumn{2}{|c|}{0.764} & \multicolumn{2}{|c|}{$\frac{1}{0.227}$} & \multicolumn{2}{|c|}{0.070} & \multicolumn{2}{|c|}{$\frac{1}{0.579}$} & \multicolumn{2}{|c|}{0.077} & \multicolumn{2}{|c|}{$<0.001$} \\
\hline
\end{tabular}


Age ranged from 14 to 70 years, and there were no statistically significant differences between patients with and without allergic rhinitis $(p=0.642)$

Nasal obstruction intensity was evaluated in both groups. Most of the patients had moderate to severe complaints in the groups, with no statistically significant differences between them.

As for the presence of preoperative snoring, it was found that most of group I patients snored (29 [74.4\%]), whereas nine patients in group II $(50 \%)$ snored, with no statistically significant differences $(p=0.07)$.

Regarding the presence of facial pressure, 22 patients had the symptom, 16 in group I (41\%) and six in group II (33.3\%). These numbers did not show statistically significant differences.

The presence of smell alterations was also assessed. Most patients with anosmia/hyposmia (13) were from group I. Only two patients in group II had this alteration. However, these results showed no statistically significant differences.

Operating time was assessed in both groups during surgery (Fig. 5). There were statistically significant differences between the groups, as group I had a longer operation time, on average $(p=0.001)$.

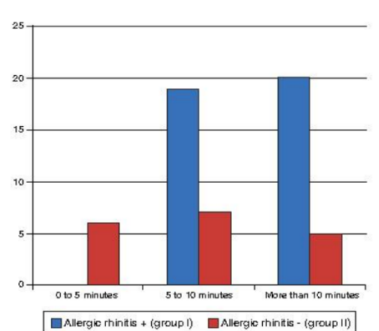

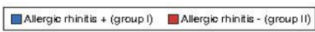

Figure 5 Operative time.

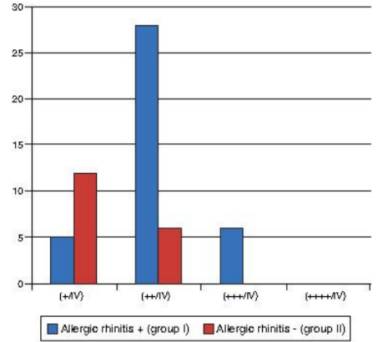

OAllerge thinitis + (group 1) DAllerge rinitits - (group 11)
Bleeding was also evaluated intraoperatively, which tended to be higher in group I (most degrees + and +++/IV) than in group II (most degrees + and $++/$ IV) with statistically significant differences $(p<$ 0.001), as shown in Fig. 6.

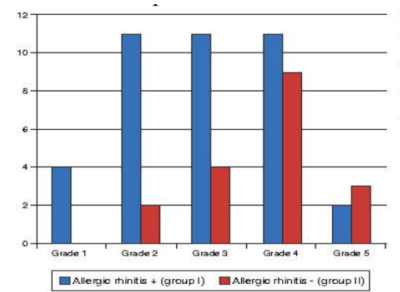

Figure 6 Transoperative bleeding.

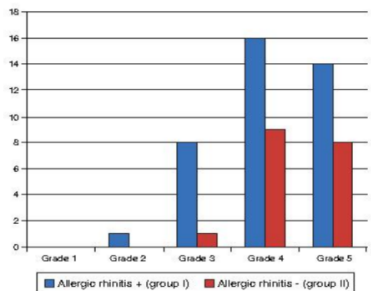

All patients were assessed postoperatively on days seven, 30, and 90 . On the seventh day after the surgery, patients were assessed in relation to breathing. There were no statistically significant differences between groups $(p=0.079)$. The prevalence was higher at grades II, III, and IV (Fig. 7). Improvement was not expected in all patients at this phase, due to swelling and presence of crusts, but many patients already mentioned improvement.

Figure 7 Degree of breathing improvement - seventh day postoperatively.

On the 30th day after surgery, the degree of improvement in breathing was evaluated again and there were still no statistically significant differences between groups $(p=0.271)$, as shown in Fig. 8. Most patients reported improvements at grades IV and V.

Figure 8 Degree of breathing improvement - 30th day postoperatively.

On the 90th day, the degree of improvement in breathing was reassessed (Fig. 9). This evaluation showed considerable improvement of results, as usually there are no more crusts and edema. ${ }^{18}$ There were no statistically significant differences between the groups $(p=0.808)$.
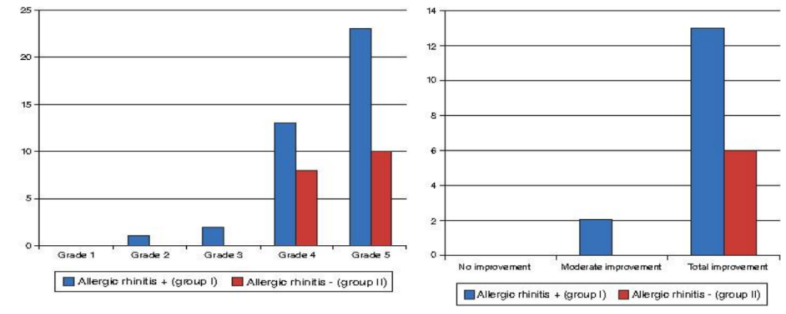

Figure 9 Degree of breathing improvement -90th day postoperatively.

Regarding the improvement in the smell alteration symptom, all patients showed improvement after the operation. Only two patients in group I showed mild improvement, whereas all others achieved complete improvement (Fig. 10). There were no statistically significant differences ( $p=1.000$ - Fisher's exact test).

\section{Figure 10 Degree of olfaction improvement.}

Regarding the facial pressure variable, most patients achieved complete improvement with surgery. Only one patient did not achieve any improvement, as shown in Fig. 11. There were no statistically significant differences ( $p=1.000$ - Fisher's exact test).
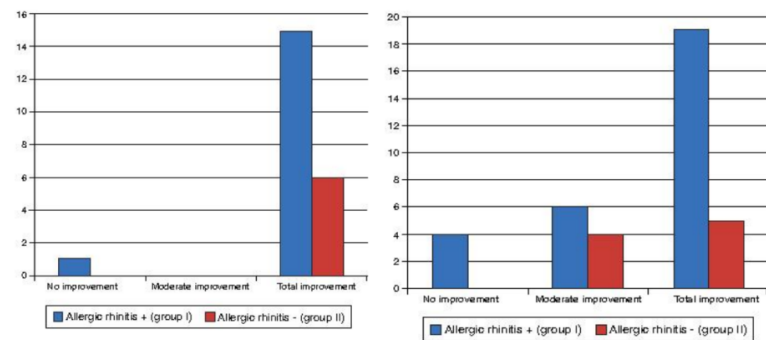

Figure 11 Degree of facial pressure improvement.

Regarding the snoring, of the 38 patients who snored, most showed moderate or complete improvement, as shown in Fig. 12. There were no statistically significant differences between groups $(p=0.588)$

\section{Figure 12 Degree of snoring improvement.}

Finally, on the 90th day postoperatively, improvement in symptoms of sneezing, itching, and rhinorrhea was assessed in group I. Of the 39 patients, only four did not show any improvement, as shown in Fig. 13.

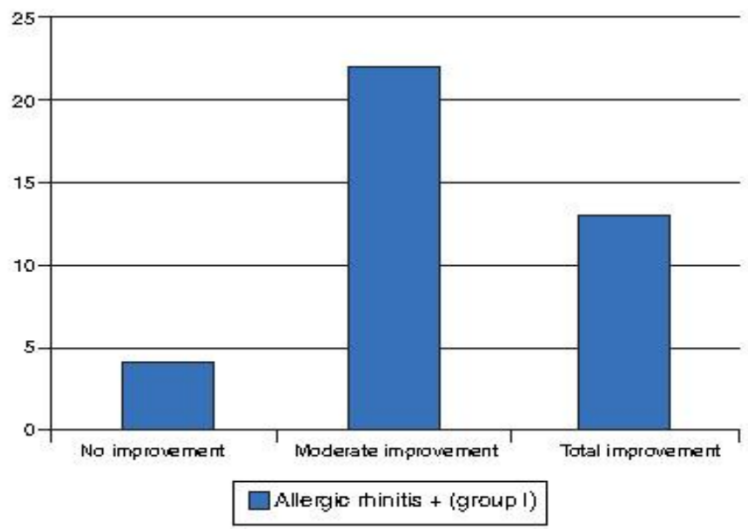

Figure 13 Degree of improvement in sneezing, pruritus, and rhinorrhea.

\section{CONCLUSION}

This study demonstrated the efficacy of inferior turbinoplasty three months after the surgery regarding non-obstructive symptoms of snoring, anosmia, facial pressure, itching, sneezing, and rhinorrhea, in addition to obstructive symptoms in patients with and without allergic rhinitis.

\section{REFERENCES}

1. Passali D, Passali FM, Damiani V, Passali GC, Bellussi L. Treatment of inferior turbinate hypertrofy: a randomized clinical trial. Ann Otol Rhinol Laryngol. 2003;112:683-8.

2. Mello Junior JFD, Mion O. Rinite alérgica. In: Campos Ch, Olival Ho, editors. Tratado 
de otorrinolaringologia, vol. III, 2nd ed. São Paulo: Roca; 2011.

3. Solé D, Mello Júnior JF, Weckx LLM, Rosário Filho NA. III Consenso sobre rinites. Braz J Mtorinolary JF, Weck LL

4. Batra PS, Seiden AM, Smith TL. Surgical management of adult inferior turbinate hypertrophy. Laryngoscope. 2009;119:1819-27.

5. Puterman MM, Segal N, Joshua BZ. Endoscopic, assisted, modified turbinoplasty with mucosal flap. J Laryngol Otol. 2012;126:525-8.

6. El Henawi D, Ahmer MR, Madian YT. Comparison between power-assisted turbinoplasty and submucosal resection in the treatment of inferior turbinate hypertrophy. ORL J Otorhino- laryngol Relat Spec. 2011;73:151-5.

7. Brandarkar ND, Smith TL. Outcomes of surgery for inferior turbinate hypertrophy. Curr Opin Otolaryngol Head Neck Surg. 2010;18:49-53.

8. Montovani JC, Gomes MA, Balbani APS. Sintomas de distúrbios do sono em pacientes com rinite. Rev Bras Alergia Imunopatol. 2008;31:249-52.

9. Guilemany JM, Garcia- Pinerõ A, Alobid I, Cardelús S, Centellas S, Bartra J, et al Guilemany JM, Garcia- Pinerõ A, Alobid I, Cardelus S, Centellas S, Bartra J, et al.
Persistent allergic rhinitis has a moderate impact in the sense of smell, depending on both nasal congestion and inflammation. Laryngoscope. 2009;119:233-8. 